TITLE:

\title{
Quark beta decay in the inhomogeneous chiral phase and cooling of compact stars
}

\author{
$\operatorname{AUTHOR}(\mathrm{S}):$ \\ Tatsumi, T.; Muto, T.
}

CITATION:

Tatsumi, T. ...[et al]. Quark beta decay in the inhomogeneous chiral phase and cooling of compact stars. Physical Review D 2014, 89(10): 103005.

ISSUE DATE:

2014-05

URL:

http://hdl.handle.net/2433/187783

RIGHT:

(C) 2014 American Physical Society 
PHYSICAL REVIEW D 89, 103005 (2014)

\title{
Quark beta decay in the inhomogeneous chiral phase and cooling of compact stars
}

\author{
T. Tatsumi ${ }^{1}$ and T. Muto ${ }^{2}$ \\ ${ }^{1}$ Department of Physics, Kyoto University, Kyoto 606-8502, Japan \\ ${ }^{2}$ Department of Physics, Chiba Institute of Technology, \\ 2-1-1 Shibazono, Narashino, Chiba 275-0023, Japan
}

(Received 8 March 2014; published 8 May 2014)

\begin{abstract}
A novel cooling mechanism is proposed for neutron stars, based on the recent development in the studies of the QCD phase diagram. A possible appearance of the inhomogeneous chiral phase makes the quark beta decay without gluonic interaction. An estimate of the neutrino emissivity shows the order of $10^{24-26}\left(T / 10^{9}\right)^{6}\left(\mathrm{erg} \mathrm{cm}^{-3} \mathrm{~s}^{-1}\right)$ near the phase boundaries, whose efficiency is comparable with the usual quark cooling or pion cooling, but it works only in the limited density region. These features may give another cooling scenario of neutron stars.
\end{abstract}

DOI: $10.1103 /$ PhysRevD.89.103005

PACS numbers: 21.65.Qr, 26.60.Kp, 97.60.Gb

\section{INTRODUCTION}

The appearance of the inhomogeneous phases near the phase boundary should be a rather common phenomenon in many-body systems. The Fulde-Ferrell-Larkin-Ovchinikov state is one of the typical examples in superconductivity in the presence of magnetic impurities [1] and has recently been studied in dilute atomic gas [2] or within the context of color superconductivity in QCD [3]. Inhomogeneous phase formation in magnetic materials is another one: spin density wave $[4,5]$ or texture [6]. A similar subject has also been addressed in the QCD phase diagram. The deconfinement and chiral transition have been studied both theoretically and experimentally in the QCD phase diagram [7]. The direct numerical calculation based on the lattice QCD theory should be a most powerful tool for this purpose, but its validity is, for the present, limited to high temperature and low density regions due to the sign problem. On the contrary, the phase structure is also important and interesting in the high-density region in the light of recent progress in the observation of compact stars [8]. Many theoretical studies have been devoted to the chiral transition by the use of the effective models of QCD [7]. Consequently, spontaneous symmetry breaking should be restored at high density, which is specified by the vanishment of the $q \bar{q}$ scalar condensate, $\langle\bar{\psi} \psi\rangle$ : it is the order parameter in the chiral transition and takes a finite value in the vacuum to generate the quark or nucleon mass. In these studies it is implicitly assumed that the condensate is scalar and uniform, while Lorenz invariance or parity symmetry no longer holds at finite densities.

Recently there appeared many papers about the possibility of the inhomogeneous chiral phases [9], where the condensates are not restricted to the scalar one and they are spatially nonuniform, stimulated by the mathematical discoveries of the Hartree-Fock solutions in the $1+1$ dimensions [10]; it has been shown that analytic solutions are obtained in terms of the elliptic functions in the GrossNeveu model or two-dimensional Nambu-Jona-Lasinio (NJL) model in the large $N$ limit. The order parameter or the mean field is generalized to be complex as $M(x)=\langle\bar{\psi} \psi\rangle+i\left\langle\bar{\psi} i \gamma_{5} \psi\right\rangle=\Delta(x) e^{i \theta(x)}$, and Baser and Dunne have found the solutions of the self-consistent coupled equations of quark and $M(x)$ for these models. Its direct application is possible for the one-dimensional order in $1+3$ dimensions by embedding the onedimensional structure and operating the Lorentz boost in the direction perpendicular to it. Actually Nickel has performed this procedure for the real kink crystal (RKC) [11], where $\theta(\mathbf{r})=0$. A similar procedure may also be possible for the chiral spiral. The chiral spiral has a history. Nakano and one of the authors (T. T.) have studied the possibility of the inhomogeneous chiral phase in $(1+3)$ dimensional quark matter within the $S U(2) \times S U(2) \mathrm{NJL}$ model [12]. Using $\theta(\mathbf{r})=\mathbf{q} \cdot \mathbf{r}$, the chiral condensates take form, $\langle\bar{\psi} \psi\rangle=\Delta \cos (\mathbf{q} \cdot \mathbf{r}),\left\langle\bar{\psi} i \gamma_{5} \tau_{3} \psi\right\rangle=\Delta \sin (\mathbf{q} \cdot \mathbf{r})$, which is a $(1+3)$-dimensional realization of the chiral spiral in $1+1$ dimensions. They called it dual-chiraldensity wave (DCDW). Since the spatial displacement of the condensates is compensated by chiral rotation on the quark field, the external degrees of freedom are mixed with the internal ones; the wave function changes $\psi \rightarrow e^{i \mathbf{k} \cdot \mathbf{d}} \exp \left(i \gamma_{5} \tau_{3} \mathbf{q} \cdot \mathbf{d} / 2\right) \psi$ following the displacement, $\mathbf{r} \rightarrow \mathbf{r}+\mathbf{d}$.

The physical mechanism has been discussed in Ref. [12]; the nesting effect of the Fermi surface may play a key role as in condensed matter physics [4,5,13-15]. If this is the case, the appearance of the inhomogeneous phase should be rather robust and less model dependent. However, there are still many subjects to be elucidated. In Ref. [11] Nickel suggested that RKC is more favorite than DCDW in symmetric quark matter in the chiral limit by comparing the thermodynamic potential. However, it should be an ideal situation, and we must carefully compare both cases in 
realistic situations, including the model dependence $[16,17]$. In particular, the effect of the quark current mass $[18,19]$ and magnetic field should be important [20,21]. Actually chiral anomaly plays an important role and DCDW develops in a wide region in the presence of the magnetic field [20,21]. Asymmetric quark matter or chemical equilibrium is also important in compact stars [22]. Thus more elaborate studies are needed to say definite things about the most plausible configuration, the critical density, or the critical temperature. Besides, the relation to other phases such as color superconductivity [23] or the extension to the $S U(3)$ case [24] should be an interesting subject.

On the other hand, it should be important to consider their phenomenological implications. Since the order parameter is spatially nonuniform and takes a periodic function, one may expect elasticity like a Coulomb lattice or liquid crystal [25]. The periodicity of the order parameter may give rise to another effect. The quark wave function accordingly takes a special form dictated by the generalized Bloch theorem [10]; momentum is not a good quantum number, so the condensates should modify the momentum conservation in the elementary processes like the Umklapp process in a solid [26]. Moreover, the appearance of the pseudoscalar condensate is related to magnetic properties $[12,21]$. Thus it should be interesting and important to figure out how such features manifest by confronting them with physical phenomena. In the relativistic heavy-ion collisions the formation of a quark-gluon plasma has been expected. Some implication of the chiral critical point has been studied theoretically and experimentally [7]. If the inhomogeneous phases are realized during the collisions, they might give rise to some phenomena never discussed yet [27]. In this paper we consider the cooling process in compact stars as an astrophysical implication of the inhomogeneous phases.

The cooling of compact stars has provided us with information about the form of matter at high densities [28]. Recent observations of the surface temperature of young pulsars have suggested that some compact stars such as $3 \mathrm{C} 58$ or Vela seem to have rather low temperatures, which should barely be explained by the standard scenario. Such stars might require exotic cooling; quark cooling is one of the fast cooling mechanisms in the core region. On the other hand, Cas A also presents important information about the thermal evolution of young pulsars [29]. Considering the young age of $t=330 \mathrm{yr}$, the observed effective temperature of Cas A also gives a strong constraint on the equation of state and cooling processes. In a recent paper we have presented models that satisfy both cases of Cas A and other cooler stars by considering the quark matter in the core [30].

As a cooling mechanism in quark matter, the neutrino emission by way of the direct Urca process is well known and standard, $d \rightarrow u+e^{-}+\bar{\nu}_{e}, u+e^{-} \rightarrow d+\nu_{e}$ [31]. This process works for interacting quarks, while it is strongly prohibited for free and light quarks due to the kinematical condition (triangular condition) at low temperature.
The neutrino emissivity is then efficient and proportional to $\alpha_{s} T^{6}$ with the QCD coupling constant $\alpha_{s}$ [32].

Here we discuss a new cooling mechanism, based on the recent development in the understanding of the QCD phase diagram: a possible appearance of the inhomogeneous phases near the chiral transition [7]. Accordingly the chiral condensates modify the elementary process by supplying the extra momentum at the interaction vertex [33].

This paper is organized as follows. In Sec. II we present our framework for calculating the neutrino emissivity, where some characteristic aspects associated with the DCDW phase are pointed out. In Sec. III numerical results for the neutrino emissivity are demonstrated, and their implications for the cooling of hybrid stars are briefly discussed in Sec. IV. The summary and concluding remarks are given in Sec. V. Properties of the quark propagator are summarized in Appendix A. The evaluation of the weak matrix element is presented in Appendix B, and details of angular integrals for obtaining the emissivity in two limiting cases are given in Appendixes C and D.

\section{FRAMEWORK}

\section{A. DCDW}

First we briefly summarize the results about DCDW in the previous work [12]. The DCDW phase can be represented as a chirally rotated state from normal quark matter,

$$
\begin{aligned}
|\mathrm{DCDW}\rangle & \left.=\exp \left(i \int \theta(\mathbf{r}) A_{3}^{0}(\mathbf{r}) d^{3} r\right) \mid \text { normal }\right\rangle \\
& \left.\equiv U_{\mathrm{DCDW}}(\theta) \mid \text { normal }\right\rangle,
\end{aligned}
$$

where $A_{i}^{\mu}$ denotes the axial-vector current with the $i$ th isospin component. We restrict the chiral transformation to $U_{I_{3}}(1)$ around the third axis in the isospin space to preserve the electromagnetic charge of the system. Then we can easily check the following relations:

$$
\begin{aligned}
\langle\mathrm{DCDW}|\bar{\psi} \psi| \mathrm{DCDW}\rangle & =\Delta \cos (\mathbf{q} \cdot \mathbf{r}), \\
\left\langle\mathrm{DCDW}\left|\bar{\psi} i \gamma_{5} \tau_{3} \psi\right| \mathrm{DCDW}\right\rangle & =\Delta \sin (\mathbf{q} \cdot \mathbf{r}),
\end{aligned}
$$

for $\theta(\mathbf{r})=\mathbf{q} \cdot \mathbf{r}$, where the amplitude $\Delta$ is given by $\langle$ normal $|\bar{\psi} \psi|$ normal $\rangle$. In the following we use the NJL model with $S U(2) \times S U(2)$ symmetry in the chiral limit, as an effective model of QCD. When we define the new quark field $\psi_{W}$ by way of the Weinberg transformation such that

$$
\psi_{W}=\exp \left(i \gamma_{5} \tau_{3} \mathbf{q} \cdot \mathbf{r} / 2\right) \psi,
$$

$\psi_{W}$ satisfies the following Hartree equation:

$$
\left(i \not \gamma-m+1 / 2 \gamma_{5} \tau_{3} \not\right) \psi_{W}=0,
$$

with $q=(0, \mathbf{q})$. Here $m=-2 G \Delta$ is the dynamical quark mass generated by the quark-quark interaction with the 
coupling constant $G$. The quark eigenstate (quasiparticle) then can be represented by $|\mathbf{p}, \eta, \epsilon\rangle$ with quantum numbers momentum $\mathbf{p}, \eta= \pm 1$ specified by the spin polarization and $\epsilon= \pm 1$ the particle and antiparticle. Accordingly the energy eigenvalues read

$$
E_{\mathbf{p}}^{\eta}=\epsilon\left(E_{p}^{2}+|\mathbf{q}|^{2} / 4+\eta \sqrt{(\mathbf{p} \cdot \mathbf{q})^{2}+m^{2}|\mathbf{q}|^{2}}\right)^{1 / 2},
$$

with $E_{p}=\left(|\mathbf{p}|^{2}+m^{2}\right)^{1 / 2}$. Thus the Fermi surfaces of the quasiparticles are deformed in this case: one has the prolate shape and the other the oblate shape (see Figs. 1 and 2).

Choosing $\mathbf{q} / / \hat{z}$ without loss of generality, the eigenfunction renders [12]

$$
\langle\mathbf{r} \mid \mathbf{p}, \eta, \epsilon=1\rangle=u_{W}^{\eta}(\mathbf{p}) \exp (i \mathbf{p} \cdot \mathbf{r})
$$

with the spinor

$$
u_{W}^{\eta}=\left(\begin{array}{c}
a_{1}^{\eta} \phi_{+}+a_{2}^{\eta} \phi_{-} \\
b_{1}^{\eta} \phi_{+}+b_{2}^{\eta} \phi_{-}
\end{array}\right),
$$

where $\phi_{ \pm}$is the Pauli spinor being specified by $\sigma_{z} \phi_{ \pm}=$ $\pm \phi_{ \pm}$and the coefficients $a_{i}^{\eta}, b_{i}^{\eta}$ are given by

$$
\begin{gathered}
\frac{a_{1}^{\eta}}{a_{2}^{\eta}}=\frac{p_{-}}{p_{z}} \cdot \frac{m+\eta \beta}{E_{\mathbf{p}}^{\eta}-|\mathbf{q}| / 2-\eta \beta}, \\
\frac{b_{1}^{\eta}}{a_{2}^{\eta}}=\frac{p_{-}}{E_{\mathbf{p}}^{\eta}-|\mathbf{q}| / 2-\eta \beta}, \\
\frac{b_{2}^{\eta}}{a_{2}^{\eta}}=\frac{p_{z}}{-m+\eta \beta},
\end{gathered}
$$

for $\tau_{3}=1$ and $q_{z}=|\mathbf{q}|$, with $\beta \equiv\left(p_{z}^{2}+m^{2}\right)^{1 / 2}$ and $p_{-} \equiv$ $p_{1}-i p_{2}$ for the positive-energy solutions. The negativeenergy solutions $(\epsilon=-1)$ are obtained by replacing $E_{\mathbf{p}}^{\eta}$ by $-E_{\mathbf{p}}^{\eta}$. Note that these eigenfunctions are written in terms of the newly defined quark field $\psi_{W}$.

DCDW develops between the onset chemical potential $\mu_{c 1}$ and the termination one $\mu_{c 2}$. Their values and those of the parameters are listed in Table I [12].

\section{B. Neutrino emissivity}

We consider the neutrino emissivity in the presence of DCDW, following Refs. [35],[36],[37]. Consider the beta decay of $d$ quarks, $d\left(p_{1}\right) \rightarrow u\left(p_{2}\right)+e^{-}\left(p_{3}\right)+\bar{\nu}_{e}\left(p_{4}\right)$ in the DCDW phase, where $p_{i}=\left(E_{i}, \mathbf{p}_{i}\right)$ denotes the

TABLE I. Values of the chemical potentials and the parameters in the unit of the cutoff parameter $\Lambda=850 \mathrm{MeV}$.

\begin{tabular}{lccccc}
\hline \hline$\mu_{c 1}$ & $\mu_{c 2}$ & $m_{c 1}$ & $m_{c 2}$ & $|\mathbf{q}|_{c 1}$ & $|\mathbf{q}|_{c 2}$ \\
\hline 0.49 & 0.53 & 0.2 & 0.01 & 0.55 & 0.8 \\
\hline \hline
\end{tabular}

four-momentum. Taking the effective interaction as the current-current form, $H_{W}=\frac{\tilde{G}_{F}}{\sqrt{2}} h_{1+i 2}^{\mu} l_{\mu}+$ H.c., the transition matrix element is given as $W_{f i} \equiv\left\langle u, e^{-}, \bar{\nu}_{e}\left|H_{W}\right| d\right\rangle=$ $\left\langle u_{W}, e^{-}, \bar{\nu}_{e}\left|\tilde{H}_{W}\right| d_{W}\right\rangle$, where

$\tilde{H}_{W}=U_{\mathrm{DCDW}}(\mathbf{q}) H_{W} U_{\mathrm{DCDW}}^{\dagger}(\mathbf{q})=\frac{\tilde{G}_{F}}{\sqrt{2}} \tilde{h}_{1+i 2}^{\mu} l_{\mu}+$ H.c.

$\tilde{G}_{F}=G_{F} \cos \theta_{C}$ with $G_{F}$ being the Fermi weak coupling constant and $\theta_{C}$ the Cabibbo angle. Here it is to be noted that the matrix element between eigenstates $|\mathbf{p}, \eta, \epsilon\rangle$ should be calculated by using the untransformed states, $\exp \left(-i \gamma_{5} \tau_{3} \mathbf{q} \cdot \mathbf{r} / 2\right)|\mathbf{p}, \eta, \epsilon\rangle$, as in the pion cooling [35]. The transformed quark current $\tilde{h}_{1+i 2}^{\mu}$ now reads

$$
\tilde{h}_{1+i 2}^{\mu}=U_{\mathrm{DCDW}}(\mathbf{q}) h_{1+i 2}^{\mu} U_{\mathrm{DCDW}}^{\dagger}(\mathbf{q})=\exp (-i \mathbf{q} \cdot \mathbf{r}) h_{1+i 2}^{\mu},
$$

by way of the current algebra, which implies that DCDW modifies the momentum conservation at the weakinteraction vertex [38]. The usual triangular condition among $\mathbf{p}_{1}, \mathbf{p}_{2}$, and $\mathbf{p}_{3}$ is now relaxed by the momentum supply from DCDW, so that the beta decay process becomes possible.

Since quarks should be treated as quasiparticles in our case, the naive application of the emissivity based on the Fermi golden rule is not relevant: we must properly take into account the wave-function renormalization besides the deformation of the Fermi surface. Thus we start with a more general formula. The neutrino emissivity can be then given as $[39,40]$

$$
\begin{aligned}
\epsilon= & N_{c} \tilde{G}_{F}^{2} \int \frac{d^{3} p_{3}}{(2 \pi)^{3} 2 E_{3}} \int \frac{d^{3} p_{4}}{(2 \pi)^{3} 2 E_{4}} E_{4} L_{\lambda \sigma} \\
& \times n_{F}\left(-E_{3}+\mu_{e}\right) f_{B}\left(k_{0}\right) \operatorname{Im} \Pi_{R}^{\lambda \sigma}(k),
\end{aligned}
$$

with $k=\left(E_{3}+E_{4}-\mu_{e}, \mathbf{p}_{3}+\mathbf{p}_{4}\right)$, the Fermi-Dirac distribution function $n_{F}$ and the Bose-Einstein one $f_{B}$. The information of the quark tensor is summarized in the $W$ boson polarization tensor,

$$
\Pi_{R}^{\lambda \sigma}(k)=T \sum_{n} \int \frac{d^{3} p_{1}}{(2 \pi)^{3}} \operatorname{tr}\left[\Gamma^{\lambda} S_{W}^{d}\left(p_{1}\right) \Gamma^{\sigma} S_{W}^{u}\left(p_{1}-k \pm q\right)\right]
$$

with $\Gamma^{\mu}=\gamma^{\mu}\left(1-\gamma_{5}\right)$ and the quark propagator, $S_{W}^{-1}(p)=\not p-m+\gamma_{5} \tau_{3} q / 2$. Since the contribution from the Dirac sea is small at low temperature and high density, the quark thermal Green's function approximately renders

$$
S_{W}^{i} \simeq \sum_{\eta= \pm} \frac{\rho_{i}^{\eta}}{i \omega_{n}-\left(E_{p}^{\eta}-\mu_{i}\right)},
$$

in terms of the density matrices $\rho_{i}^{ \pm}$(see Appendix A), where $\omega_{n}$ denotes the Matsubara frequency, $\omega_{n}=(2 n+1) \pi T$. After some manipulation, we have an expression for the emissivity, 


$$
\begin{aligned}
\epsilon_{\mathrm{DCDW}}= & 2 N_{c} V^{-1}\left[\prod_{i=1}^{4} V \int \frac{d^{3} p_{i}}{(2 \pi)^{3}}\right] E_{4} W_{f i} n_{F}\left(\mathbf{p}_{1}\right) \\
& \times\left(1-n_{F}\left(\mathbf{p}_{2}\right)\right)\left(1-n_{F}\left(\mathbf{p}_{3}\right)\right),
\end{aligned}
$$

where $W_{f i}$ is the transition rate for the beta decay of the $d$ quark in the DCDW phase.

\section{Transition rate}

The transition rate is given as

$W_{f i}=V(2 \pi)^{4} \delta^{(4)}\left(p_{1}-p_{2}-p_{3}-p_{4} \pm q\right)|M|^{2} / \prod_{i=1}^{4}\left(2 E_{i} V\right)$

with

$$
|M|^{2}=\frac{1}{2} \sum_{\sigma_{1}, \sigma_{2}, \sigma_{3}}\left|M_{f i}\right|^{2}
$$

where the squared matrix element can be evaluated as

$$
\begin{aligned}
\left|M_{f i}\right|^{2} & =\frac{\tilde{G}_{F}^{2}}{2} \operatorname{tr}\left(\rho_{e} \Gamma_{\mu} \rho_{\nu_{e}} \Gamma_{\nu}\right) \operatorname{tr}\left(\rho_{u} \Gamma^{\mu} \rho_{d} \Gamma^{\nu}\right) \\
& \equiv \frac{\tilde{G}_{F}^{2}}{2} H^{\mu \nu} L_{\mu \nu}
\end{aligned}
$$

in terms of the density matrices, $\rho_{i}, i=u, d$ for quarks, and

$$
\begin{aligned}
& \rho_{e}=\not \rho_{3}+m_{e}, \\
& \rho_{\nu}=\not p_{4},
\end{aligned}
$$

for leptons. Note that the sum over the spin polarizations of quarks is taken in Eq. (17). The leptonic tensor $L_{\mu \nu}$ can easily be evaluated as

$$
\begin{aligned}
L_{\mu \nu} & =\sum_{\sigma_{3}} \operatorname{tr}\left(\rho_{e} \Gamma_{\mu} \rho_{\nu_{e}} \Gamma_{\nu}\right) \\
& =8\left[p_{3 \mu} p_{4 \nu}-g_{\mu \nu} p_{3} p_{4}+p_{3 \nu} p_{4 \mu}+i \epsilon_{\alpha \mu \beta \nu} p_{3}^{\alpha} p_{4}^{\beta}\right] .
\end{aligned}
$$

The quark tensor $H^{\alpha \beta}$ has a somewhat complicated form. Consider

$$
H_{\eta \eta^{\prime}}^{\mu \nu} \equiv \frac{1}{4} \operatorname{tr}\left(\Lambda_{u}^{\eta} \Gamma^{\mu} \Lambda_{d}^{\eta^{\prime}} \Gamma^{\nu}\right)
$$

for the spin polarization $\eta, \eta^{\prime}(= \pm 1)$ by the use of the density matrices $\Lambda^{ \pm}$in Appendix A. The evaluation of the quark tensor is straightforward to give

$$
H_{\eta \eta^{\prime}}^{\mu \nu}=2\left[k_{1}^{\eta, \mu} k_{2}^{\eta^{\prime}, \nu}-g^{\mu \nu} k_{1}^{\eta} k_{2}^{\eta^{\prime}}+k_{1}^{\eta, \nu} k_{2}^{\eta^{\prime}, \mu}+i \epsilon^{\alpha \mu \beta \nu} k_{1 \alpha}^{\eta} k_{2 \beta}^{\eta^{\prime}}\right]
$$

(Appendix B), where $k_{i}^{\eta}$ is defined as

$$
\begin{aligned}
& k_{1}^{\eta}=\left(p_{1}^{\eta}-\frac{q}{2}\right)\left(1-p_{1}^{\eta} Q_{1}^{\eta}\right)+m^{2} Q_{1}^{\eta}, \\
& k_{2}^{\eta^{\prime}}=\left(p_{2}^{\eta^{\prime}}+\frac{q}{2}\right)\left(1-p_{2}^{\eta^{\prime}} Q_{2}^{\eta^{\prime}}\right)+m^{2} Q_{2}^{\eta^{\prime}},
\end{aligned}
$$

with $Q_{i}^{\eta}=-\eta q / \sqrt{\left(p_{i}^{\eta} q\right)^{2}-m^{2} q^{2}}$ and $p_{i}^{\eta}=\left(E_{i}^{\eta}, \mathbf{p}_{i}\right)$, where $\eta$ and $\eta^{\prime}$ denote the spin polarization for the $d$ quark and $u$ quark, respectively. Then

$$
\begin{aligned}
\left|M_{f i}^{\eta \eta^{\prime}}\right|^{2} & =\frac{\tilde{G}_{F}^{2}}{2} H_{\eta \eta^{\prime}}^{\mu \nu} L_{\mu \nu} \\
& =32 \tilde{G}_{F}^{2}\left(k_{2}^{\eta^{\prime}} p_{3}\right)\left(k_{1}^{\eta} p_{4}\right),
\end{aligned}
$$

which is reduced to $32 \tilde{G}_{F}^{2}\left(p_{2} p_{3}\right)\left(p_{1} p_{4}\right)$ as $q \rightarrow 0$. After summing over $\sigma_{2}$ and averaging over $\sigma_{1}$, we immediately get the Iwamoto's result [32].

On the other hand,

$$
\begin{aligned}
& k_{1}^{\eta} \rightarrow\left(p_{1}^{\eta}-\frac{q}{2}\right)\left(1-\eta \frac{\mathbf{p}_{1} \cdot \mathbf{q}}{\left|\mathbf{p}_{1} \cdot \mathbf{q}\right|}\right), \\
& k_{2}^{\eta^{\prime}} \rightarrow\left(p_{2}^{\eta^{\prime}}+\frac{q}{2}\right)\left(1+\eta^{\prime} \frac{\mathbf{p}_{2} \cdot \mathbf{q}}{\left|\mathbf{p}_{2} \cdot \mathbf{q}\right|}\right),
\end{aligned}
$$

in the massless limit. If $\mathbf{q}$ is taken as the $z$ direction, only the half-space is relevant for each momentum integral, depending on $\eta$. We shall see the neutrino emission is prohibited in this case, irrespective of $\mathbf{q}$, by the energy-momentum conservation unless the interaction is not included, as in the direct URCA process. We recall that the driving mechanism for the emergence of DCDW is the level splitting by the mass term between the energy spectra of massless quarks with relative momentum difference $\mathbf{q}$. Also, since the mass is proportional to the amplitude of DCDW, there should not be left any effect in the massless limit.

\section{PHASE SPACE INTEGRAL}

Taking $[35,41]$ for references, we try to manipulate the phase space integral for the emissivity (15). The energymomentum conservation reads

$$
\begin{gathered}
\mathbf{p}_{1}=\mathbf{p}_{2}+\mathbf{p}_{3}+\mathbf{p}_{4}+\mathbf{q}, \\
E_{1}^{\eta}=E_{2}^{\eta^{\prime}}+E_{3}+E_{4} .
\end{gathered}
$$

Dropping $\mathbf{p}_{4}$ in Eq. (26) because of $\left|\mathbf{p}_{4}\right|=O(T)$,

$\left(\mathbf{p}_{1}-\mathbf{q} / 2\right)^{2} \simeq\left(\mathbf{p}_{2}+\mathbf{q} / 2\right)^{2}+\left|\mathbf{p}_{3}\right|^{2}+2 \mathbf{p}_{3}\left(\mathbf{p}_{2}+\mathbf{q} / 2\right)$,

which is recast into 
QUARK BETA DECAY IN THE INHOMOGENEOUS CHIRAL ...

PHYSICAL REVIEW D 89, 103005 (2014)

$$
\begin{aligned}
&\left(E_{1}^{\eta}\right)^{2}-\left(\eta \sqrt{\left(\mathbf{p}_{1} \cdot \mathbf{q}\right)^{2}+m^{2}|\mathbf{q}|^{2}}+\mathbf{q} \cdot \mathbf{p}_{1}\right) \\
& \simeq\left(E_{2}^{\eta^{\prime}}\right)^{2}-\left(\eta^{\prime} \sqrt{\left(\mathbf{p}_{2} \cdot \mathbf{q}\right)^{2}+m^{2}|\mathbf{q}|^{2}}-\mathbf{q} \cdot \mathbf{p}_{2}\right) \\
& \quad+\left|\mathbf{p}_{3}\right|^{2}+2 \mathbf{p}_{3}\left(\mathbf{p}_{2}+\mathbf{q} / 2\right),
\end{aligned}
$$

where we put $m_{u} \simeq m_{d} \equiv m$. Similarly, we find

$$
\left(E_{1}^{\eta}\right)^{2} \simeq\left(E_{2}^{\eta^{\prime}}\right)^{2}+E_{3}^{2}+2 E_{2}^{\eta^{\prime}} E_{3},
$$

from Eq. (27) by neglecting $E_{4}$ again. From Eqs. (29) and (30) we have

$$
\begin{aligned}
(\eta & \left.\sqrt{\left(\mathbf{p}_{1} \cdot \mathbf{q}\right)^{2}+m^{2}|\mathbf{q}|^{2}}+\mathbf{q} \cdot \mathbf{p}_{1}\right) \\
& -\left(\eta^{\prime} \sqrt{\left(\mathbf{p}_{2} \cdot \mathbf{q}\right)^{2}+m^{2}|\mathbf{q}|^{2}}-\mathbf{q} \cdot \mathbf{p}_{2}\right) \\
& +2 \mathbf{p}_{3}\left(\mathbf{p}_{2}+\mathbf{q} / 2\right) \simeq 2 E_{3} E_{2}^{\eta^{\prime}},
\end{aligned}
$$

where we used $E_{3} \simeq\left|\mathbf{p}_{3}\right|$.

\section{A. Case of the massless-quark limit}

First, we consider the massless limit by setting $m=0$. Equation (31) is then reduced to a simple one,

$$
\begin{aligned}
& \eta\left|\mathbf{p}_{1} \cdot \mathbf{q}\right|\left(1+\eta \frac{\mathbf{p}_{1} \cdot \mathbf{q}}{\left|\mathbf{p}_{1} \cdot \mathbf{q}\right|}\right)-\eta^{\prime}\left|\mathbf{p}_{2} \cdot \mathbf{q}\right|\left(1-\eta^{\prime} \frac{\mathbf{p}_{2} \cdot \mathbf{q}}{\left|\mathbf{p}_{2} \cdot \mathbf{q}\right|}\right) \\
& \quad+2 \mathbf{p}_{3}\left(\mathbf{p}_{2}+\mathbf{q} / 2\right) \simeq 2 E_{3} E_{2}^{\eta^{\prime}} .
\end{aligned}
$$

The first two terms should be vanished for the nonzero value of $k_{i}^{\alpha}$ from Eq. (25). Using Eq. (25), the squared matrix element (24) is also reduced to a simple one,

$$
\begin{aligned}
\left|M_{f i}^{\eta \eta^{\prime}}\right|^{2}= & 32 \tilde{G}_{F}^{2}\left(k_{1}^{\eta} p_{4}\right)\left(1+\eta^{\prime} \frac{\mathbf{p}_{2} \cdot \mathbf{q}}{\left|\mathbf{p}_{2} \cdot \mathbf{q}\right|}\right) \\
& \times\left(E_{p_{2}}^{\eta^{\prime}} E_{3}-\left(\mathbf{p}_{2}+\mathbf{q} / 2\right) \cdot \mathbf{p}_{3}\right),
\end{aligned}
$$

which gives no contribution due to Eqs. (25) and (32). Generally the emissivity is vanished as $\mathbf{q}$ or $m$ goes to zero as it should be.

Since the Fermi surface is well deformed as the wave vector $\mathbf{q}$ increases [12], the general expression of the emissivity is difficult to be evaluated. However, one may estimate it by considering the specific cases near the phase boundaries of the DCDW phase, where the deformation is very weak at one side and extremely strong at the other side.

\section{B. Near the onset density of DCDW}

\section{Effective Fermi sphere}

First, we consider the cooling rate near the onset density of the DCDW phase, where the deformation of the Fermi surface is not so remarkable (Fig. 1). So, one may introduce the effective

where $p_{z}^{\max } \simeq \sqrt{\left(\mu_{i}+|\mathbf{q}| / 2\right)^{2}-m^{2}}$ for each $u$ or $d$ quark. Thus the radius of the effective Fermi sphere is given as 


$$
\bar{p}_{F i} \simeq\left(\mu_{i}+|\mathbf{q}| / 2\right)\left[\frac{4 \mu_{i}-|\mathbf{q}|}{4 \mu_{i}+2|\mathbf{q}|}\right]^{1 / 3} \simeq \mu_{i}+|\mathbf{q}| / 4+\cdots,
$$

where we have used $\mu_{i} \gg|\mathbf{q}| / 2 \simeq m$. Note that the quark energy is now approximated as $E_{\mathbf{p}}^{ \pm} \simeq|\mathbf{p}|$ within the same approximation. In the following we evaluate the emissivity by assuming massless quarks in the presence of DCDW. Usually it vanishes in the absence of DCDW by the kinematical conditions. Following Iwamoto [32], we begin with the formula,

$$
\left|M_{f i}^{\eta \eta^{\prime}}\right|^{2}=32 \tilde{G}_{F}^{2}\left(p_{1} \cdot p_{4}\right)\left(p_{2} \cdot p_{3}\right)
$$

for the squared matrix element. Note that the factor 2 is different from [32] since only the one polarization is relevant. It can be further written as

$\left|M_{f i}^{\eta \eta^{\prime}}\right|^{2} \simeq 32 \tilde{G}_{F}^{2} E_{1} E_{2} E_{3} E_{4}\left(1-\cos \theta_{14}\right)\left(1-\cos \theta_{23}\right)$,

where $\theta_{14}\left(\theta_{23}\right)$ is the angle between momenta of the $d$ quark and neutrino (the angle between momenta of the $u$ quark and electron).

\section{Expression of the emissivity}

Setting the momentum magnitudes of quarks and electrons equal to their values on the respective Fermi surfaces, one obtains

$$
\begin{aligned}
\epsilon_{\mathrm{DCDW}} \simeq & \frac{3}{(2 \pi)^{8}} \bar{p}_{F u}^{2} \bar{p}_{F d}^{2} \mu_{e}^{2} \int d E_{1} d E_{2} d E_{3} E_{4}^{2} d E_{4} \delta\left(E_{1}-E_{2}-E_{3}-E_{4}\right) \prod_{i=1}^{3}\left(2 E_{i}\right)^{-1} \\
& \times n\left(\mathbf{p}_{1}\right)\left[1-n\left(\mathbf{p}_{2}\right)\right]\left[1-n\left(\mathbf{p}_{3}\right)\right]\left(\prod_{i=1}^{4} \int d \Omega_{i}\right)\left|M_{f i}^{\eta \eta^{\prime}}\right|^{2} \delta^{(3)}\left(\mathbf{p}_{1}-\mathbf{p}_{2}-\mathbf{p}_{3}-\mathbf{p}_{4}-\mathbf{q}\right),
\end{aligned}
$$

where only one polarization has been taken into account for the initial $d$ quarks. First we can proceed with the angular integral as in the pion-condensed case [35],

$$
A \equiv \prod_{i=1}^{4}\left(\int d \Omega_{i}\right)\left|M_{f i}^{\eta \eta^{\prime}}\right|^{2} \delta^{(3)}\left(\mathbf{p}_{1}-\mathbf{p}_{2}-\mathbf{p}_{3}-\mathbf{p}_{4}-\mathbf{q}\right) .
$$

In the following, the neutrino momentum $\mathbf{p}_{4}$ in the delta function is dropped because of $\left|\mathbf{p}_{\bar{\nu}}\right|=O(T)$. After integrating with respect to the angle $\Omega_{4}$ with the squared matrix element (37), one has

$$
A \simeq \frac{32}{2 \pi^{2}} \tilde{G}_{F}^{2} E_{1} E_{2} E_{3} E_{4} \tilde{A},
$$

where

$$
\begin{aligned}
\tilde{A} & =\left(\prod_{i=1}^{3} \int d \Omega_{i}\right)\left(1-\cos \theta_{23}\right) \int d^{3} x e^{i\left(\mathbf{p}_{1}-\mathbf{p}_{2}-\mathbf{p}_{3}-\mathbf{q}\right) \cdot \mathbf{x}} \\
& \simeq \frac{64 \pi^{5}}{\left|\mathbf{p}_{1}\right|\left|\mathbf{p}_{2}\right||\mathbf{q}|} .
\end{aligned}
$$

The derivation of $\tilde{A}$ is given in Appendix C. Note that this integral gives a finite value only if the triangle condition is satisfied in the limit $|\mathbf{q}| \rightarrow 0$ as shown in Appendix C. The remaining phase-space integration in Eq. (38) leads to the emissivity,

$$
\epsilon_{\mathrm{DCDW}} \simeq \frac{3}{(2 \pi)^{5}} \mu_{u} \mu_{d} \frac{\mu_{e}^{2}}{2|\mathbf{q}|} 32 \tilde{G}_{F}^{2} I,
$$

with

$$
\begin{aligned}
I= & \left(\prod_{i=1}^{3} \int_{-\infty}^{\infty} d E_{i}\right) \int_{0}^{\infty} d E_{4} E_{4}^{3} \delta\left(E_{1}-E_{2}-E_{3}-E_{4}\right) \\
& \times n\left(\mathbf{p}_{1}\right)\left[1-n\left(\mathbf{p}_{2}\right)\right]\left[1-n\left(\mathbf{p}_{3}\right)\right]=\frac{457}{5040} \pi^{6} T^{6} .
\end{aligned}
$$

The emissivity of the neutrino process, $u+e^{-} \rightarrow d+\nu_{e}$, gives the same contribution as that of the process, $d \rightarrow u+e^{-}+\bar{\nu}_{e}$. Therefore, by multiplying a factor 2 , one finally has

$$
\epsilon_{\mathrm{DCDW}} \simeq \frac{457}{1680} \pi \tilde{G}_{F}^{2} \mu_{u} \mu_{d} \frac{\mu_{e}^{2}}{|\mathbf{q}|} T^{6}
$$

Assuming $\mu_{u}=\mu_{d}$, as in the noninteracting $u, d$ quark matter, and using the values in Table I, we can estimate its numerical value as

$$
\epsilon_{\mathrm{DCDW}} \simeq 6.1 \times 10^{26}\left(\rho_{B} / \rho_{0}\right)^{2 / 3} Y_{e}^{2 / 3} T_{9}^{6}\left(\mathrm{ergcm}^{-3} \mathrm{~s}^{-1}\right),
$$

where $Y_{e}$ is the electron number fraction in quark matter, $Y_{e}=\rho_{e} / \rho_{B}, \quad \rho_{0}$ is the nuclear saturation density, $\rho_{0} \simeq 0.17 \mathrm{fm}^{-3}$, and $T_{9} \equiv T / 10^{9}(\mathrm{~K})$.

\section{Near the termination density of DCDW}

\section{Deformation of the Fermi surface}

Near the termination density the dynamical quark mass is also very small while the wave vector $\mathbf{q}$ is still large. The quark energy is well approximated as $E_{p}^{-} \simeq|\mathbf{p} \pm \mathbf{q} / 2|$ for major quarks: the Fermi seas are then remarkably deformed to be almost separated spheres with centers shifted by $\mathbf{q}$ (see Fig. 2). Using Eq. (31), we have the squared matrix element (24), 


$$
\begin{aligned}
\left|M_{f i}^{\eta \eta^{\prime}}\right|^{2} \simeq & 32 \tilde{G}_{F}^{2}\left[\{ E _ { 1 } E _ { 4 } - ( \mathbf { p } _ { 1 } - \mathbf { q } / 2 ) \cdot \mathbf { p } _ { 4 } \} \left\{\frac{\eta}{2} \frac{m^{2}|\mathbf{q}|^{2}}{\sqrt{\left(\mathbf{p}_{1} \cdot \mathbf{q}\right)^{2}+m^{2}|\mathbf{q}|^{2}}}\left(1+\eta^{\prime} \frac{\mathbf{\mathbf { p } _ { 2 } \cdot \mathbf { q }}}{\sqrt{\left(\mathbf{p}_{2} \cdot \mathbf{q}\right)^{2}+m^{2}|\mathbf{q}|^{2}}}\right)\right.\right. \\
& \left.-\eta^{\prime} \frac{m^{2}\left(\mathbf{p}_{3}+\mathbf{q} / 2\right) \cdot \mathbf{q}}{\sqrt{\left(\mathbf{p}_{2} \cdot \mathbf{q}\right)^{2}+m^{2}|\mathbf{q}|^{2}}}\left(1-\eta \frac{\mathbf{p}_{1} \cdot \mathbf{q}}{\sqrt{\left(\mathbf{p}_{1} \cdot \mathbf{q}\right)^{2}+m^{2}|\mathbf{q}|^{2}}}\right)\right\}+\frac{1}{2} m^{2}\left(\mathbf{p}_{4} \cdot \mathbf{q}\right)\left(1+\eta \frac{\mathbf{q}}{\sqrt{\left(\mathbf{p}_{1} \cdot \mathbf{q}\right)^{2}+m^{2}|\mathbf{q}|^{2}}}\right) \\
& \left.\times\left(1+\eta^{\prime} \frac{\mathbf{p}_{2} \cdot \mathbf{q}}{\sqrt{\left(\mathbf{p}_{2} \cdot \mathbf{q}\right)^{2}+m^{2}|\mathbf{q}|^{2}}}\right)-\eta \eta^{\prime} \frac{m^{4}\left(\mathbf{p}_{3}+\mathbf{q} / 2\right) \cdot \mathbf{q}\left(\mathbf{p}_{4} \cdot \mathbf{q}\right)}{\sqrt{\left(\mathbf{p}_{1} \cdot \mathbf{q}\right)^{2}+m^{2}|\mathbf{q}|^{2}} \sqrt{\left(\mathbf{p}_{2} \cdot \mathbf{q}\right)^{2}+m^{2}|\mathbf{q}|^{2}}}\right]
\end{aligned}
$$

with the proper momentum restriction. Note that it has obviously vanished when $m \rightarrow 0$ or $|\mathbf{q}| \rightarrow 0$.

Next consider the angular integrations of the squared matrix element. Since one spin polarization is dominant, it is sufficient to consider only the case where $\eta=\eta^{\prime}=-1$ for $u, d$ quarks.

We first perform the angular integration in (15),

$$
B=\left(\prod_{i=1}^{4} \int d \Omega_{i}\right)\left|M_{f i}^{\eta \eta^{\prime}}\right|^{2} \delta^{3}\left(\mathbf{p}_{1}-\mathbf{p}_{2}-\mathbf{p}_{3}-\mathbf{p}_{4}-\mathbf{q}\right) .
$$

Near the termination density, $m \ll\left|\mathbf{p}_{i}\right| \simeq \mu_{i}<|\mathbf{q}|(i=u, d)$ (see Table I). Thereby we make an approximation to neglect the terms $m^{2}|\mathbf{q}|^{2}$ appearing in the denominators in the four parentheses $(\cdots)$ in Eq. (46). Furthermore we drop $\mathbf{p}_{4}$ from the delta function in Eq. (47) since $\left|\mathbf{p}_{4}\right|=O(T)$. Then, substituting the matrix element (46) into (47) and after integrating with respect to the angle $\Omega_{4}$, one obtains

$$
\begin{aligned}
B= & \frac{32}{2 \pi^{2}} \tilde{G}_{F}^{2} m^{2} E_{1}^{\eta} E_{4}\left(\prod_{i=1}^{3} \int d \Omega_{i}\right) \int d^{3} x e^{i\left(\mathbf{p}_{1}-\mathbf{p}_{2}-\mathbf{p}_{3}-\mathbf{q}\right) \cdot \mathbf{x}}\left[\frac { | \mathbf { q } | ^ { 2 } } { 2 } \left\{-\frac{1}{\sqrt{\left(\mathbf{p}_{1} \cdot \mathbf{q}\right)^{2}+m^{2}|\mathbf{q}|^{2}}}\left(1-\frac{\mathbf{p}_{2} \cdot \mathbf{q}}{\left|\mathbf{p}_{2} \cdot \mathbf{q}\right|}\right)\right.\right. \\
& \left.\left.+\frac{1}{\sqrt{\left(\mathbf{p}_{2} \cdot \mathbf{q}\right)^{2}+m^{2}|\mathbf{q}|^{2}}}\left(1+\frac{\mathbf{p}_{1} \cdot \mathbf{q}}{\left|\mathbf{p}_{1} \cdot \mathbf{q}\right|}\right)\right\}+\frac{\mathbf{p}_{3} \cdot \mathbf{q}}{\sqrt{\left(\mathbf{p}_{2} \cdot \mathbf{q}\right)^{2}+m^{2}|\mathbf{q}|^{2}}}\left(1+\frac{\mathbf{p}_{1} \cdot \mathbf{q}}{\left|\mathbf{p}_{1} \cdot \mathbf{q}\right|}\right)\right] .
\end{aligned}
$$

The available range of the momenta $\mathbf{p}_{1}$ and $\mathbf{p}_{2}$ contributing to $B$ is such that $\mathbf{p}_{1} \cdot \mathbf{q}>0$ and $\mathbf{p}_{2} \cdot \mathbf{q}<0$, which result from $\left(1+\mathbf{p}_{1} \cdot \mathbf{q} /\left|\mathbf{p}_{1} \cdot \mathbf{q}\right|\right)>0$ and $\left(1-\mathbf{p}_{2} \cdot \mathbf{q} /\left|\mathbf{p}_{2} \cdot \mathbf{q}\right|\right)>0$ in Eq. (48). As illustrated in Fig. 3, only the $d$ quarks occupying the upper part of the "two-center" Fermi surface and $u$ quarks occupying the lower part of the two-center Fermi surface contribute to the reaction. Changing the variables $\mathbf{p}_{1}, \mathbf{p}_{2}$ to the new ones,

$$
\mathbf{p}_{1}^{\prime} \equiv \mathbf{p}_{1}-\mathbf{q} / 2, \quad \mathbf{p}_{2}^{\prime} \equiv \mathbf{p}_{2}+\mathbf{q} / 2,
$$

we have, from Eq. (5),

$$
E_{1}^{\eta} \simeq\left|\mathbf{p}_{1}^{\prime}\right|, \quad E_{2}^{\eta^{\prime}} \simeq\left|\mathbf{p}_{2}^{\prime}\right|,
$$

which means both the angular integrations with respect to $\mathbf{p}_{i}^{\prime}(i=1,2)$ have spherical symmetry. In terms of the new variables $\mathbf{p}_{1}^{\prime}, \mathbf{p}_{2}^{\prime}, B$ is rewritten as

$$
B=\frac{32}{2 \pi^{2}} \tilde{G}_{F}^{2} m^{2} E_{1}^{\eta} E_{4}\left(\tilde{B}_{1}+\tilde{B}_{2}+\tilde{B}_{3}\right),
$$

where

$$
\begin{aligned}
& \tilde{B}_{1}=-\left(\prod_{i=1}^{3} \int d \Omega_{i}\right) \int d^{3} x \exp \left[i\left(\mathbf{p}_{1}^{\prime}-\mathbf{p}_{2}^{\prime}-\mathbf{p}_{3}\right) \cdot \mathbf{x}\right] \frac{|\mathbf{q}|^{2}}{\sqrt{\left\{\left(\mathbf{p}_{1}^{\prime}+\mathbf{q} / 2\right) \cdot \mathbf{q}\right\}^{2}+m^{2}|\mathbf{q}|^{2}}} \simeq-32 \pi^{5} \frac{|\mathbf{q}|}{\left|\mathbf{p}_{1}^{\prime}\right|^{2}\left|\mathbf{p}_{2}^{\prime}\right|\left|\mathbf{p}_{3}\right|} \log \left(\frac{2|\mathbf{q}|}{m}\right), \\
& \tilde{B}_{2}=\left(\prod_{i=1}^{3} \int d \Omega_{i}\right) \int d^{3} x \exp \left[i\left(\mathbf{p}_{1}^{\prime}-\mathbf{p}_{2}^{\prime}-\mathbf{p}_{3}\right) \cdot \mathbf{x}\right] \frac{|\mathbf{q}|^{2}}{\sqrt{\left\{\left(\mathbf{p}_{2}^{\prime}-\mathbf{q} / 2\right) \cdot \mathbf{q}\right\}^{2}+m^{2}|\mathbf{q}|^{2}}} \simeq 32 \pi^{5} \frac{|\mathbf{q}|}{\left|\mathbf{p}_{1}^{\prime}\right|\left|\mathbf{p}_{2}^{\prime}\right|^{2}\left|\mathbf{p}_{3}\right|} \log \left(\frac{2|\mathbf{q}|}{m}\right),
\end{aligned}
$$

$$
\begin{aligned}
\tilde{B}_{3} & =\left(\prod_{i=1}^{3} \int d \Omega_{i}\right) \int d^{3} x \exp \left[i\left(\mathbf{p}_{1}^{\prime}-\mathbf{p}_{2}^{\prime}-\mathbf{p}_{3}\right) \cdot \mathbf{x}\right] \frac{2\left(\mathbf{p}_{3} \cdot \mathbf{q}\right)}{\sqrt{\left\{\left(\mathbf{p}_{2}^{\prime}-\mathbf{q} / 2\right) \cdot \mathbf{q}\right\}^{2}+m^{2}|\mathbf{q}|^{2}}} \\
& \simeq 16 \pi^{5}|\mathbf{q}| \frac{\left|\mathbf{p}_{1}^{\prime}\right|^{2}-\left|\mathbf{p}_{2}^{\prime}\right|^{2}-\left|\mathbf{p}_{3}\right|^{2}}{\left|\mathbf{p}_{1}^{\prime}\right|\left|\mathbf{p}_{2}^{\prime}\right|^{4}\left|\mathbf{p}_{3}\right|}\left[\log \left(\frac{2|\mathbf{q}|}{m}\right)-2\right] .
\end{aligned}
$$



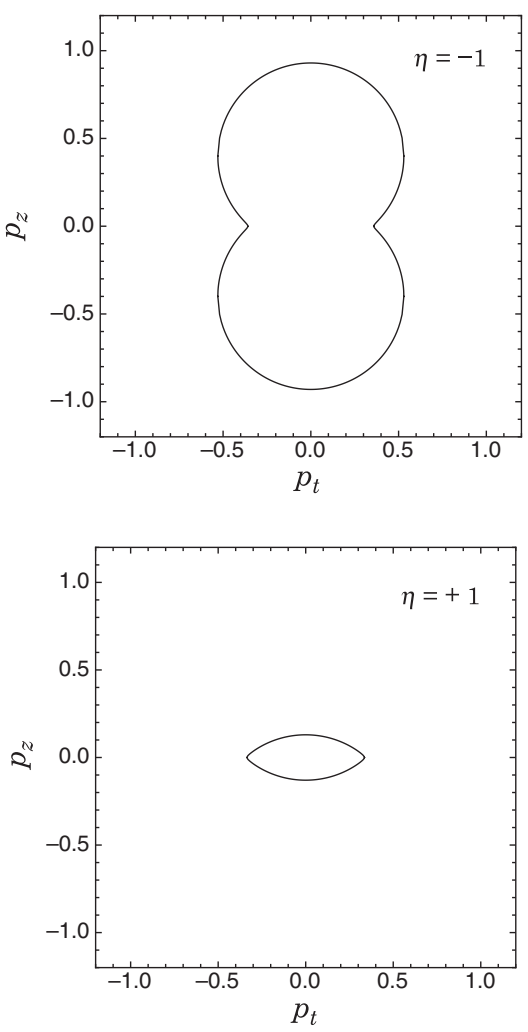

FIG. 2. Fermi surfaces at the termination density with anarbitrary scale. The meaning of the legend and symbols are the same as in Fig. 1.
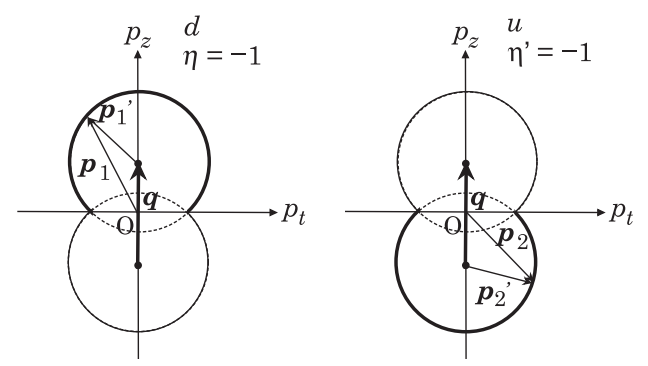

FIG. 3. Schematic view of the Fermi surfaces for $d$ quarks with $\eta=-1$ and $u$ quarks with $\eta^{\prime}=-1$ near the termination density. See the text for the meaning of the legend and symbols.

The details of evaluating the $\tilde{B}_{i}(i=1-3)$ are shown in Appendix D.

\section{Expression of the emissivity}

By the use of Eqs. (51) and (52) for the angular integral $B$, the emissivity [Eq. (15)] is written in the case of the region near the termination density as

$$
\begin{aligned}
\epsilon_{\mathrm{DCDW}} \simeq & \frac{3}{(2 \pi)^{8}}\left|\mathbf{p}_{F u}\right|^{2}\left|\mathbf{p}_{F d}\right|^{2} \mu_{e}^{2} \int d E_{1} d E_{2} d E_{3} E_{4}^{2} d E_{4} \\
& \times \delta\left(E_{1}-E_{2}-E_{3}-E_{4}\right) \prod_{i=1}^{3}\left(2 E_{i}\right)^{-1} \\
& \times n\left(\mathbf{p}_{1}\right)\left[1-n\left(\mathbf{p}_{2}\right)\right]\left[1-n\left(\mathbf{p}_{3}\right)\right] B,
\end{aligned}
$$

where $\left|\mathbf{p}_{F i}\right|(i=u, d)$ is the Fermi momentum of the quark. Noting that $\left|\mathbf{p}_{1}^{\prime}\right| \sim E_{1},\left|\mathbf{p}_{2}^{\prime}\right| \sim E_{2},\left|\mathbf{p}_{3}\right| \sim E_{3},\left|\mathbf{p}_{4}\right|=E_{4}$ and by the use of Eq. (43), one can perform the remaining phase-space integrations in (53). With the help of the chemical equilibrium relation,

$$
\mu_{d}=\mu_{u}+\mu_{e},
$$

and by multiplying a factor of 2 to take into account the neutrino process, $u+e^{-} \rightarrow d+\nu_{e}$, one obtains the final expression for the emissivity,

$$
\begin{aligned}
\epsilon_{\mathrm{DCDW}}= & \frac{1}{2} \frac{457}{1680} \pi \tilde{G}_{F}^{2} m^{2}|\mathbf{q}| \frac{\mu_{e}}{\mu_{u}} T^{6} \\
& \times\left\{\log \left(\frac{2|\mathbf{q}|}{m}\right)+\frac{\mu_{d}}{\mu_{u}}\left[\log \left(\frac{2|\mathbf{q}|}{m}\right)-2\right]\right\} .
\end{aligned}
$$

Assuming again $\mu_{u} \simeq \mu_{d}$ in the $u, d$ quark matter, and using the values in Table I, we can estimate the numerical value,

$$
\epsilon_{\mathrm{DCDW}}=\left(2.16 \times 10^{24}\right)\left(\frac{\rho_{\mathrm{B}}}{\rho_{0}}\right)^{1 / 3} Y_{e}^{1 / 3} T_{9}^{6} \quad\left(\mathrm{erg} \mathrm{cm}^{-3} \mathrm{~s}^{-1}\right) .
$$

\section{DISCUSSION}

In both regions near the onset density (I) and near the termination density (II), the wave vector $\mathbf{q}$, which marks the inhomogeneity of the DCDW phase, plays an essential role in the enhancement of neutrino emissions via the quark beta decay. Owing to the existence of $\mathbf{q}$, there is no need to supply energy and momentum to the reactions through spectator particles. As a result, the available phase space for the quark beta decay in the DCDW phase is enough to give a large neutrino emissivity, which is proportional to $T^{6}$, as is the case with other exotic cooling mechanisms [32,35-37].

The neutrino emissivity in the DCDW phase near the onset density, $\epsilon_{\mathrm{DCDW}}^{(\mathrm{I})}$, is proportional to $1 /|\mathbf{q}|$ [Eq. (44)]. This $\mathbf{q}$ dependence originates from the angular integral of the phase factor, $\exp (-i \mathbf{q} \cdot \mathbf{x})$ in (41), and such specific momentum dependence is similar to that in the pioncondensed case, where the neutrino emissivity, given on the basis of spherical Fermi surfaces for baryons, is proportional to $1 /|\mathbf{k}|$ with $|\mathbf{k}|$ being the momentum of the $p$-wave pion condensates $[35,36]$. The magnitude of $\epsilon_{\text {DCDW }}^{(\mathrm{I})}[\mathrm{Eq} .(45)]$ is of the same order as those for normal quark cooling [32] and pion cooling [35,36]. On the other hand, the neutrino emissivity in the DCDW phase near the termination density, $\epsilon_{\mathrm{DCDW}}^{(\mathrm{II})}$, has a complicated dependence on $|\mathbf{q}|$ and $m$ including the terms $\propto m^{2}|\mathbf{q}| \log (2|\mathbf{q}| / m)$ [Eq. (55)], which reflects a singular structure of the quark tensor originating from the deformed Fermi surface [see Figs. 2 and 3]. The resulting emissivity $\epsilon_{\mathrm{DCDW}}^{(\mathrm{II})}$ is smaller than $\epsilon_{\mathrm{DCDW}}^{(\mathrm{I})}$ by 2 orders of magnitude [see Eqs. (45) and 
(56)] but is still larger than emissivities for standard cooling processes such as the modified Urca process by a factor of $\sim 10^{3} / T_{9}^{2}$ [42].

It should be noted that the enhancement of neutrino emissions works in the limited density region, because the DCDW phase appears only near the chiral transition. Only the shell region of the radius width $\Delta R$ inside hybrid stars is responsible to the fast cooling mechanism. If enhanced, the cooling region is limited only to such inhomogeneous phases; heavier compact stars may not necessarily cool faster than lighter ones. This opens up another possibility for explaining the thermal evolution of Cas $\mathrm{A}$ and other cooler stars in a consistent way, as recently proposed by Noda et al. based on the model separating the quark matter region into the color-flavor-locked phase and the nonsuperconducting quark phase [30].

\section{SUMMARY AND CONCLUDING REMARKS}

We have proposed a novel cooling mechanism (DCDW cooling) of hybrid stars, based on the idea of the inhomogeneous chiral phase. It originates from the nonperturbative effect of QCD at moderate densities. We have shown that the beta decay process becomes possible in the DCDW phase due to the momentum supply by DCDW at the weak-interaction vertex. The emissivity is estimated near the phase boundaries of the DCDW phase to be the order of $10^{24-26} T_{9}^{6}\left(\mathrm{erg} \mathrm{cm}^{-3} \mathrm{~s}^{-1}\right)$, whose value may be comparable with that by the quark cooling [32] or pion cooling [35,36]. Another important point is that this mechanism works in the limited density region where the DCDW phase appears. This feature is similar to the Cooper pair-breaking-formation process, where the limited density region is efficient in the superfluid phase [43].

If we incorporate this mechanism in the calculation of the cooling curves of young neutron stars, further works are needed that consider the realistic equation of state of cold catalyzed quark matter instead of flavor symmetric quark matter and the numerical values of emissivity over the whole region of the DCDW phase without the restriction to the phase boundaries [44]. The effects of the magnetic field are also an interesting subject, since there should be a large magnetic field inside compact stars. The appearance of DCDW appears to be robust and less model dependent in the presence of the magnetic field [20,21,45].

In this paper we considered the DCDW phase as a typical inhomogeneous chiral phase, but the similar mechanism may be possible for other configurations such as RKC.

It is also interesting to seek other phenomenological implications of the inhomogeneous chiral phases by considering their elasticity [46] or magnetic properties.

\section{ACKNOWLEDGMENTS}

The authors thank T. Maruyama, N. Yasutake, and T. Noda for their interest in this work. This work is partially supported by the Grant-in-Aid for Scientific Research on Innovative Areas through No. 24105008 provided by MEXT and the Grant-in-Aid for Scientific Research (C) (No. 23540318) by JSPS.

\section{APPENDIX A: QUARK PROPAGATOR IN THE DCDW PHASE}

The quark propagator is given by

$$
S_{W}^{i}(p)=\frac{1}{\not p-m+\gamma_{5} \tau_{3} \not 2} \equiv \frac{N^{i}}{D},
$$

with $i=u, d$ for $\tau_{3}= \pm 1$, respectively, in the DCDW phase within the mean-field approximation [12], where the index $W$ indicates that we define the new quark field by way of the Weinberg transformation from the original one,

$$
\psi_{W} \equiv \exp \left(i \gamma_{5} \tau_{3} \mathbf{q} \cdot \mathbf{r} / 2\right) \psi
$$

The numerator $N^{i}$ is

$$
\begin{aligned}
N^{i}= & \left(\not p+m-\gamma_{5} \tau_{3} \not 2\right)\left(p^{2}-m^{2}+q^{2} / 4\right. \\
& \left.-(p q-m \not)) \gamma_{5} \tau_{3}\right),
\end{aligned}
$$

and the denominator $D$ is

$$
D=\left(p^{2}-m^{2}+q^{2} / 4\right)^{2}-\left((p q)^{2}-m^{2} q^{2}\right) .
$$

The solutions for $D=0$, which is a transcendental equation, give the four energies corresponding to positive and negative solutions with two polarizations $\eta= \pm 1$ : the positive energy solutions are given by

$$
E_{p}^{ \pm}=\sqrt{E_{p}^{2}+|\mathbf{q}|^{2} / 4 \pm \sqrt{(\mathbf{p} \cdot \mathbf{q})^{2}+m^{2}|\mathbf{q}|^{2}}}
$$

with $E_{p}=\left(m^{2}+|\mathbf{p}|^{2}\right)^{1 / 2}$.

The density matrix for the positive-energy state is then given as

$$
\begin{aligned}
\rho_{i}^{ \pm} & =\left.\operatorname{Res} S_{W}^{i}(p)\right|_{p_{0}=E_{p}^{ \pm}} \\
& =\frac{\left.\left(\not p+m-\gamma_{5} \tau_{3} \not / 2\right)\right|_{p_{0}=E_{p}^{ \pm}}\left(1 \pm \hat{s}(p) \tau_{3}\right)}{4 E_{p}^{ \pm}} \\
& \equiv \frac{\Lambda_{i}^{ \pm}}{4 E_{p}^{ \pm}},
\end{aligned}
$$

with $\hat{s}(p) \equiv(\mathbf{p} \cdot \mathbf{q}+m q) \gamma_{5} / \sqrt{(\mathbf{p} \cdot \mathbf{q})^{2}+m^{2}|\mathbf{q}|^{2}}, \quad \hat{s}^{2}(p)=1$. We can easily check

$$
\sum_{p_{0}=E_{p}^{ \pm}} \operatorname{Res} S_{W}^{i}(p) \rightarrow \frac{\not p+m}{2 E_{p}},
$$

as should be in the limit, $\mathbf{q} \rightarrow 0$. Thus the $i$ quark propagator can be written as 


\section{T. TATSUMI AND T. MUTO}

$$
S_{W}^{i} \simeq \sum_{\eta= \pm} \frac{\rho_{i}^{\eta}}{i \omega_{n}-\left(E_{p}^{\eta}-\mu_{i}\right)},
$$

once only the positive-energy state is relevant.

\section{APPENDIX B: QUARK TENSOR}

We calculate the quark tensor $H_{\eta \eta^{\prime}}^{\mu \nu}$, which is given by

$$
H_{\eta \eta^{\prime}}^{\mu \nu}=\frac{1}{4} \operatorname{tr}\left[\Lambda_{u}^{\eta^{\prime}} \gamma^{\mu}\left(1-\gamma_{5}\right) \Lambda_{d}^{\eta} \gamma^{\nu}\left(1-\gamma_{5}\right)\right] .
$$

From Eq. (A6) in Appendix A, the density matrix $\Lambda_{i}^{ \pm}$for quark $(i=u, d)$ is written as

$$
\Lambda_{i}^{\eta}=\left(p_{i}^{\eta}+m-\gamma_{5} \frac{\tau_{3}}{2} q\right)\left[1+\eta \frac{\left(\mathbf{p}_{i} \cdot \mathbf{q}+m q\right) \gamma_{5} \tau_{3}}{\sqrt{\left(\mathbf{p}_{i} \cdot \mathbf{q}\right)^{2}+m^{2}|\mathbf{q}|^{2}}}\right],
$$

where $\eta= \pm 1$, and $\tau_{3}=1\left(\tau_{3}=-1\right)$ for $i=u(i=d)$. The four-vectors, $p_{i}^{\eta}$ and $q$, are represented as $p_{i}^{\eta}=\left(E_{p_{i}}^{\eta}, \mathbf{p}_{i}\right)$ and $q^{\alpha}=(0, \mathbf{q})$, respectively. After manipulation with the Dirac matrices, one obtains

$$
\begin{aligned}
\Lambda_{i}^{\eta}= & \left(m+A_{i}^{\eta}-\frac{1}{2} \frac{\eta m}{\sqrt{\left(\mathbf{p}_{i} \cdot \mathbf{q}\right)^{2}+m^{2}|\mathbf{q}|^{2}}} \not \not \emptyset\right) \\
& +\left(B_{i}^{\eta}+\ell_{i}^{\eta}+\eta \frac{m \tau_{3} \not p_{i}^{\eta} \not}{\sqrt{\left(\mathbf{p}_{i} \cdot \mathbf{q}\right)^{2}+m^{2}|\mathbf{q}|^{2}}}\right) \gamma_{5},
\end{aligned}
$$

where $A_{i}^{\eta}=\gamma^{\mu}\left(A_{i}^{\eta}\right)_{\mu}, \ell_{i}^{\eta}=\gamma^{\mu}\left(C_{i}^{\eta}\right)_{\mu}$, and

$$
\begin{gathered}
A_{i}^{\eta} \equiv p_{i}^{\eta}+\eta \frac{1}{2} q \frac{\mathbf{p}_{i} \cdot \mathbf{q}}{\sqrt{\left(\mathbf{p}_{i} \cdot \mathbf{q}\right)^{2}+m_{i}^{2}|\mathbf{q}|^{2}}}, \\
B_{i}^{\eta} \equiv \eta m \frac{\mathbf{p}_{i} \cdot \mathbf{q} \tau_{3}}{\sqrt{\left(\mathbf{p}_{i} \cdot \mathbf{q}\right)^{2}+m^{2}|\mathbf{q}|^{2}}} \\
C_{i}^{\eta} \equiv \eta p_{i}^{\eta} \frac{\mathbf{p}_{i} \cdot \mathbf{q} \tau_{3}}{\sqrt{\left(\mathbf{p}_{i} \cdot \mathbf{q}\right)^{2}+m^{2}|\mathbf{q}|^{2}}}+\eta \frac{m^{2} q \tau_{3}}{\sqrt{\left(\mathbf{p}_{i} \cdot \mathbf{q}\right)^{2}+m^{2}|\mathbf{q}|^{2}}} \\
+\frac{\tau_{3}}{2} q,
\end{gathered}
$$

Substitution of Eq. (B3) into $\Lambda_{u}^{\eta^{\prime}}$ and $\Lambda_{d}^{\eta}$ on the righthand side of Eq. (B1) leads to

$$
H_{\eta \eta^{\prime}}^{\mu \nu}=\frac{1}{2} \operatorname{tr}\left[\left(\mathcal{A}_{u}^{\eta^{\prime}}+\ell_{u}^{\eta^{\prime}}\right) \gamma^{\mu}\left(\AA_{d}^{\eta}+\ell_{d}^{\eta}\right) \gamma^{\nu}\left(1-\gamma_{5}\right)\right] .
$$

By the use of the formulas, $\operatorname{tr}\left(\gamma^{\mu} \gamma^{\nu} \gamma^{\rho} \gamma^{\sigma}\right)=4\left(g^{\mu \nu} g^{\rho \sigma}-\right.$ $\left.g^{\mu \rho} g^{\nu \sigma}+g^{\mu \sigma} g^{\nu \rho}\right), \operatorname{tr}\left(\gamma^{\mu} \gamma^{\nu} \gamma^{\rho} \gamma^{\sigma} \gamma^{5}\right)=-4 i \epsilon^{\mu \nu \rho \sigma}$, where $\epsilon^{0123}=$ $-\epsilon_{0123}=+1$, one finally obtains
PHYSICAL REVIEW D 89, 103005 (2014)

$$
\begin{aligned}
H_{\eta \eta^{\prime}}^{\mu \nu}= & 2\left[k_{1}^{\eta, \mu} k_{2}^{\eta^{\prime}, \nu}-g^{\mu \nu} k_{1}^{\eta} k_{2}^{\eta^{\prime}}+k_{1}^{\eta, \nu} k_{2}^{\eta^{\prime}, \mu}\right. \\
& \left.+i \epsilon^{\alpha \mu \beta \nu} k_{1 \alpha}^{\eta} k_{2 \beta}^{\eta^{\prime}}\right],
\end{aligned}
$$

where

$$
\begin{aligned}
& k_{1}^{\eta}=\left(p_{1}^{\eta}-\frac{q}{2}\right)\left(1-p_{1}^{\eta} Q_{1}^{\eta}\right)+m^{2} Q_{1}^{\eta}, \\
& k_{2}^{\eta^{\prime}}=\left(p_{2}^{\eta^{\prime}}+\frac{q}{2}\right)\left(1-p_{2}^{\eta^{\prime}} Q_{2}^{\eta^{\prime}}\right)+m^{2} Q_{2}^{\eta^{\prime}},
\end{aligned}
$$

with $Q_{i}^{\eta}=-\eta q / \sqrt{\left(p_{1}^{\eta} q\right)^{2}-m^{2} q^{2}}$. The spin polarization for the $u$ quark is denoted as $\eta^{\prime}$.

\section{APPENDIX C: ANGULAR INTEGRAL: NEAR THE ONSET DENSITY OF THE DCDW}

In Appendix $\mathrm{C}$, we evaluate the angular integral $\tilde{A}$ [Eq. (41)],

$$
\tilde{A}=\left(\prod_{i=1}^{3} \int d \Omega_{i}\right)\left(1-\cos \theta_{23}\right) \int d^{3} x e^{i\left(\mathbf{p}_{1}-\mathbf{p}_{2}-\mathbf{p}_{3}-\mathbf{q}\right) \cdot \mathbf{x}} .
$$

$\tilde{A}$ is separated into two parts: $\tilde{A}=\tilde{A}_{1}+\tilde{A}_{2}$ with

$$
\tilde{A}_{1}=\left(\prod_{i=1}^{3} \int d \boldsymbol{\Omega}_{i}\right) \int d^{3} x \exp \left[i\left(\mathbf{p}_{1}-\mathbf{p}_{2}-\mathbf{p}_{3}-\mathbf{q}\right) \cdot \mathbf{x}\right]
$$

and

$$
\begin{aligned}
\tilde{A}_{2}= & -\left(\prod_{i=1}^{3} \int d \Omega_{i}\right) \cos \theta_{23} \\
& \times \int d^{3} x \exp \left[i\left(\mathbf{p}_{1}-\mathbf{p}_{2}-\mathbf{p}_{3}-\mathbf{q}\right) \cdot \mathbf{x}\right] .
\end{aligned}
$$

The $\tilde{A}_{1}$ is represented by the use of the spherical Bessel function $j_{0}(x)$ as

$$
\begin{aligned}
\tilde{A}_{1} & =\int d^{3} x e^{-i \mathbf{q} \cdot \mathbf{x}}(4 \pi)^{3} j_{0}\left(\left|\mathbf{p}_{1}\right| x\right) j_{0}\left(\left|\mathbf{p}_{2}\right| x\right) j_{0}\left(\left|\mathbf{p}_{3}\right| x\right) \\
& =(4 \pi)^{4} \int_{0}^{\infty} d x x^{2} j_{0}(|\mathbf{q}| x) j_{0}\left(\left|\mathbf{p}_{1}\right| x\right) j_{0}\left(\left|\mathbf{p}_{2}\right| x\right) j_{0}\left(\left|\mathbf{p}_{3}\right| x\right) \\
& =\frac{64 \pi^{5}}{\left|\mathbf{p}_{1}\right|\left|\mathbf{p}_{2}\right||\mathbf{q}|}
\end{aligned}
$$

for || $\mathbf{p}_{1}|-| \mathbf{p}_{2}||+\left|\mathbf{p}_{3}\right|<|\mathbf{q}|<\left|\mathbf{p}_{1}\right|+\left|\mathbf{p}_{2}\right|-\left|\mathbf{p}_{3}\right|$. This kinematical condition is met in the case near the onset density, since $\left|\mathbf{p}_{1}\right| \sim\left|\mathbf{p}_{2}\right| \sim \mu_{\mathrm{cl}}=0.49 \Lambda \gg\left|\mathbf{p}_{3}\right| \sim \mu_{e}$, and $|\mathbf{q}|=0.55 \Lambda$ (see Table I).

Next consider $\tilde{A}_{2}$. By expanding $\cos \theta_{23}$ in terms of the spherical harmonics, one has 
QUARK BETA DECAY IN THE INHOMOGENEOUS CHIRAL ...

PHYSICAL REVIEW D 89, 103005 (2014)

$$
\begin{aligned}
\tilde{A}_{2}= & -\int d^{3} x e^{-i \mathbf{q} \cdot \mathbf{x}}(4 \pi) j_{0}\left(\left|\mathbf{p}_{1}\right| x\right) \int d \Omega_{2} \int d \Omega_{3} \\
& \times \frac{4 \pi}{3} \sum_{M=-1}^{1} Y_{1}^{M *}\left(\Omega_{2}\right) Y_{1}^{M}\left(\Omega_{3}\right) e^{-i\left|\mathbf{p}_{2}\right| x \cos \theta_{2}} e^{-i\left|\mathbf{p}_{3}\right| x \cos \theta_{3}} \\
= & (4 \pi)^{4} \int_{0}^{\infty} d x x^{2} j_{0}(|\mathbf{q}| x) j_{0}\left(\left|\mathbf{p}_{1}\right| x\right) j_{1}\left(\left|\mathbf{p}_{2}\right| x\right) j_{1}\left(\left|\mathbf{p}_{3}\right| x\right),
\end{aligned}
$$

where $\theta_{i}(i=2,3)$ is the angle between $\mathbf{p}_{i}$ and $\mathbf{x}$. With $\left|\mathbf{p}_{3}\right| \sim \mu_{e} \ll \mu_{\mathrm{c} 1}$, the numerical estimation shows $\tilde{A}_{2} \ll \tilde{\tilde{A}}_{1}$, so that we can safely neglect $\tilde{A}_{2}$ in $\tilde{A}$ as compared with $\tilde{A}_{1}$.

\section{APPENDIX D: ANGULAR INTEGRAL: NEAR THE TERMINATION DENSITY OF THE DCDW}

In Appendix D, we evaluate the angular integrals, $\tilde{B}_{1}, \tilde{B}_{2}, \tilde{B}_{3}$ [Eq. (52)].

First we consider $\tilde{B}_{1}$,

$$
\begin{aligned}
\tilde{B}_{1}= & -\left(\prod_{i=1}^{3} \int d \Omega_{i}\right) \int d^{3} x \exp \left[i\left(\mathbf{p}_{1}^{\prime}-\mathbf{p}_{2}^{\prime}-\mathbf{p}_{3}\right) \cdot \mathbf{x}\right] \\
& \times \frac{|\mathbf{q}|^{2}}{\sqrt{\left[\left(\mathbf{p}_{1}^{\prime}+\mathbf{q} / 2\right) \cdot \mathbf{q}\right]^{2}+m^{2}|\mathbf{q}|^{2}}} .
\end{aligned}
$$

The angular integration over $\mathbf{p}_{2}^{\prime}$ and $\mathbf{p}_{3}$ in Eq. (D1) gives

$$
\begin{aligned}
\tilde{B}_{1}= & -4(2 \pi)^{2}|\mathbf{q}|^{2} \int d^{3} x j_{0}\left(\left|\mathbf{p}_{2}^{\prime}\right| x\right) j_{0}\left(\left|\mathbf{p}_{3}\right| x\right) \\
& \times \int d \Omega_{1} \frac{e^{i \mathbf{p}_{1}^{\prime} \cdot \mathbf{x}}}{\sqrt{\left[\left(\mathbf{p}_{1}^{\prime}+\mathbf{q} / 2\right) \cdot \mathbf{q}\right]^{2}+m^{2}|\mathbf{q}|^{2}}} .
\end{aligned}
$$

Here the factor $e^{i \mathbf{p}_{1}^{\prime} \cdot \mathbf{x}}$ can be expanded in terms of the spherical Bessel functions and the spherical harmonics as

$$
e^{i \mathbf{p}_{1}^{\prime} \cdot \mathbf{x}}=\sum_{L, M}(4 \pi) i^{L} j_{L}\left(\left|\mathbf{p}_{1}^{\prime}\right| x\right) Y_{L}^{M *}\left(\Omega_{1}\right) Y_{L}^{M}\left(\Omega_{x}\right)
$$

where the directions of $\mathbf{p}_{1}^{\prime}$ and $\mathbf{x}$ are measured from the direction of $\mathbf{q}$. Thus we can evaluate the remaining angular integrations of $\mathbf{p}_{1}^{\prime}$ and $\mathbf{x}$. By the use of the relation, $\int d \Omega_{x} Y_{L}^{M}\left(\Omega_{x}\right)=(4 \pi)^{1 / 2} \delta_{L, 0} \delta_{M, 0}$, one obtains

$$
\begin{aligned}
\tilde{B}_{1}= & -64 \pi^{3}|\mathbf{q}|^{2} \int_{0}^{\infty} d x x^{2} j_{0}\left(\left|\mathbf{p}_{1}^{\prime}\right| x\right) j_{0}\left(\left|\mathbf{p}_{2}^{\prime}\right| x\right) j_{0}\left(\left|\mathbf{p}_{3}\right| x\right) \\
& \times \int d \Omega_{1} \frac{1}{\sqrt{\left(\left|\mathbf{p}_{1}^{\prime}\right||\mathbf{q}| \cos \theta_{1}+|\mathbf{q}|^{2} / 2\right)^{2}+m^{2}|\mathbf{q}|^{2}}}
\end{aligned}
$$

In Eq. (D4),

$$
\int_{0}^{\infty} d x x^{2} j_{0}\left(\left|\mathbf{p}_{1}^{\prime}\right| x\right) j_{0}\left(\left|\mathbf{p}_{2}^{\prime}\right| x\right) j_{0}\left(\left|\mathbf{p}_{3}\right| x\right)=\frac{\pi}{4\left|\mathbf{p}_{1}^{\prime}\right|\left|\mathbf{p}_{2}^{\prime}\right|\left|\mathbf{p}_{3}\right|}
$$

for || $\mathbf{p}_{1}^{\prime}|-| \mathbf{p}_{2}^{\prime}||<\left|\mathbf{p}_{3}\right|<\left|\mathbf{p}_{1}^{\prime}\right|+\left|\mathbf{p}_{2}^{\prime}\right|$, and

$$
\begin{aligned}
\int & d \Omega_{1} \frac{1}{\sqrt{\left(\left|\mathbf{p}_{1}^{\prime}\right||\mathbf{q}| \cos \theta_{1}+|\mathbf{q}|^{2} / 2\right)^{2}+m^{2}|\mathbf{q}|^{2}}} \\
= & \frac{2 \pi}{\left|\mathbf{p}_{1}^{\prime}\right||\mathbf{q}|} I\left(\frac{|\mathbf{q}|}{2\left|\mathbf{p}_{1}^{\prime}\right|}, \frac{m}{\left|\mathbf{p}_{1}^{\prime}\right|}\right)
\end{aligned}
$$

with

$$
I(a, b) \equiv \log \left|\frac{a+1+\sqrt{(a+1)^{2}+b^{2}}}{a-1+\sqrt{(a-1)^{2}+b^{2}}}\right|
$$

Substituting Eqs. (D5), (D6), (D7) into Eq. (D4), one obtains

$$
\tilde{B}_{1} \simeq-32 \pi^{5} \frac{|\mathbf{q}|}{\left|\mathbf{p}_{1}^{\prime}\right|^{2}\left|\mathbf{p}_{2}^{\prime}\right|\left|\mathbf{p}_{3}\right|} \log \left(\frac{2|\mathbf{q}|}{m}\right)
$$

where we have used $\left|\mathbf{p}_{1}^{\prime}\right| \simeq|\mathbf{q}| / 2$ and $m \ll\left|\mathbf{p}_{1}^{\prime}\right|$.

Second, $\tilde{B}_{2}$ is calculated in a way similar to the case of the $\tilde{B}_{1}$. The result is

$$
\tilde{B}_{2} \simeq 32 \pi^{5} \frac{|\mathbf{q}|}{\left|\mathbf{p}_{1}^{\prime}\right|\left|\mathbf{p}_{2}^{\prime}\right|^{2}\left|\mathbf{p}_{3}\right|} \log \left(\frac{2|\mathbf{q}|}{m}\right)
$$

Finally, we consider $\tilde{B}_{3}$,

$$
\begin{aligned}
\tilde{B}_{3}= & \left(\prod_{i=1}^{3} \int d \Omega_{i}\right) \int d^{3} x \exp \left[i\left(\mathbf{p}_{1}^{\prime}-\mathbf{p}_{2}^{\prime}-\mathbf{p}_{3}\right) \cdot \mathbf{x}\right] \\
& \times \frac{2\left(\mathbf{p}_{3} \cdot \mathbf{q}\right)}{\sqrt{\left[\left(\mathbf{p}_{2}^{\prime}-\mathbf{q} / 2\right) \cdot \mathbf{q}\right]^{2}+m^{2}|\mathbf{q}|^{2}}}
\end{aligned}
$$

The angular integration over $\mathbf{p}_{1}^{\prime}$ gives

$$
\begin{aligned}
\tilde{B}_{3}= & 8 \pi \int d x x^{2} \int d \Omega_{x} j_{0}\left(\left|\mathbf{p}_{1}^{\prime}\right| x\right) \int d \Omega_{2} \int d \Omega_{3} \\
& \times 4 \pi \sum_{L_{2}=0}^{\infty} \sum_{M_{2}=-L_{2}}^{L_{2}}(-i)^{L_{2}} j_{L_{2}}\left(\left|\mathbf{p}_{2}^{\prime}\right| x\right) Y_{L_{2}}^{M_{2}}\left(\Omega_{2}\right) Y_{L_{2}}^{M_{2} *}\left(\Omega_{x}\right) \\
& \times 4 \pi \sum_{L_{3}=0}^{\infty} \sum_{M_{3}=-L_{3}}^{L_{3}}(-i)^{L_{3}} j_{L_{3}}\left(\left|\mathbf{p}_{3}\right| x\right) Y_{L_{3}}^{M_{3}}\left(\Omega_{3}\right) Y_{L_{3}}^{M_{3} *}\left(\Omega_{x}\right) \\
& \times \frac{\left|\mathbf{p}_{3}\right||\mathbf{q}| \cos \theta_{3}}{\sqrt{\left(\left|\mathbf{p}_{2}^{\prime}\right||\mathbf{q}| \cos \theta_{2}-|\mathbf{q}|^{2} / 2\right)^{2}+m^{2}|\mathbf{q}|^{2}}},
\end{aligned}
$$

where the remaining two exponential factors, $\exp \left(-i \mathbf{p}_{2}^{\prime} \cdot \mathbf{x}\right)$ and $\exp \left(-i \mathbf{p}_{3} \cdot \mathbf{x}\right)$ in Eq. (D10), have been expanded into terms of the spherical Bessel functions and the spherical 
harmonics. $Y_{L_{3}}^{M_{3}{ }^{*}}\left(\Omega_{x}\right)$ in Eq. (D11) is rewritten as $Y_{L_{3}}^{M_{3^{*}}}\left(\Omega_{x}\right)=(-1)^{M_{3}} Y_{L_{3}}^{-M_{3}}\left(\Omega_{x}\right)$. Then, with the help of the orthonormality of the spherical harmonics, $\int d \Omega_{x} Y_{L_{2}}^{M_{2} *}\left(\Omega_{x}\right) Y_{L_{3}}^{M_{3}}\left(\Omega_{x}\right)=\delta_{L_{2}, L_{3}} \delta_{M_{2}, M_{3}}$, Eq. (D11) reads

$$
\begin{aligned}
\tilde{B}_{3}= & 128 \pi^{3}\left|\mathbf{p}_{3}\right||\mathbf{q}| \sum_{L_{2}=0}^{\infty} \sum_{M_{2}=-L_{2}}^{L_{2}}(-1)^{L_{2}} \\
& \times \int_{0}^{\infty} d x x^{2} j_{0}\left(\left|\mathbf{p}_{1}^{\prime}\right| x\right) j_{L_{2}}\left(\left|\mathbf{p}_{2}^{\prime}\right| x\right) j_{L_{2}}\left(\left|\mathbf{p}_{3}\right| x\right) \\
& \times \int d \Omega_{2} \frac{Y_{L_{2}}^{M_{2}}\left(\Omega_{2}\right)}{\sqrt{\left(\left|\mathbf{p}_{2}^{\prime}\right||\mathbf{q}| \cos \theta_{2}-|\mathbf{q}|^{2} / 2\right)^{2}+m^{2}|\mathbf{q}|^{2}}} \\
& \times \int d \Omega_{3}(-1)^{M_{2}} Y_{L_{2}}^{-M_{2}}\left(\Omega_{3}\right) \cos \theta_{3} .
\end{aligned}
$$

The last integral in Eq. (D12) with respect to the angle of $\mathbf{p}_{3}$ yields $\sqrt{4 \pi / 3} \delta_{L_{2}, 1} \delta_{M_{2}, 0}$. Thereby Eq. (D12) reads

$$
\tilde{B}_{3}=-128 \pi^{3}\left|\mathbf{p}_{3}\right||\mathbf{q}| P Q,
$$

where

$$
\begin{aligned}
P & \equiv \int_{0}^{\infty} d x x^{2} j_{0}\left(\left|\mathbf{p}_{1}^{\prime}\right| x\right) j_{1}\left(\left|\mathbf{p}_{2}^{\prime}\right| x\right) j_{1}\left(\left|\mathbf{p}_{3}\right| x\right) \\
& =\frac{\pi}{8} \frac{\left|\mathbf{p}_{2}^{\prime}\right|^{2}+\left|\mathbf{p}_{3}\right|^{2}-\left|\mathbf{p}_{1}^{\prime}\right|^{2}}{\left|\mathbf{p}_{1}^{\prime}\right|\left|\mathbf{p}_{2}^{\prime}\right|^{2}\left|\mathbf{p}_{3}\right|^{2}}(<0),
\end{aligned}
$$

which is valid for || $\mathbf{p}_{1}^{\prime}|-| \mathbf{p}_{2}^{\prime}||<\left|\mathbf{p}_{3}\right|<\left|\mathbf{p}_{1}^{\prime}\right|+\left|\mathbf{p}_{2}^{\prime}\right|$, and

$$
\begin{aligned}
Q & \equiv \int d \Omega_{2} \frac{\cos \theta_{2}}{\sqrt{\left(\left|\mathbf{p}_{2}^{\prime}\right||\mathbf{q}| \cos \theta_{2}-|\mathbf{q}|^{2} / 2\right)^{2}+m^{2}|\mathbf{q}|^{2}}} \\
& =\frac{2 \pi}{\left|\mathbf{p}_{2}^{\prime}\right||\mathbf{q}|} J\left(\frac{|\mathbf{q}|}{2\left|\mathbf{p}_{2}^{\prime}\right|}, \frac{m}{\left|\mathbf{p}_{2}^{\prime}\right|}\right)
\end{aligned}
$$

with

$$
\begin{aligned}
J(a, b) \equiv & \left(\sqrt{(1-a)^{2}+b^{2}}-\sqrt{(1+a)^{2}+b^{2}}\right) \\
& +a I(a, b) .
\end{aligned}
$$

Since $\left|\mathbf{p}_{2}^{\prime}\right| \simeq|\mathbf{q}| / 2$ and $m \ll\left|\mathbf{p}_{2}^{\prime}\right|$, we have

$$
Q \simeq \frac{\pi}{\left|\mathbf{p}_{2}^{\prime}\right|^{2}}\left[\log \left(\frac{2|\mathbf{q}|}{m}\right)-2\right]
$$

Substituting Eqs. (D14) and (D17) into Eq. (D13), one finally obtains

$$
\begin{aligned}
\tilde{B}_{3} \simeq & 16 \pi^{5}|\mathbf{q}| \frac{\left|\mathbf{p}_{1}^{\prime}\right|^{2}-\left|\mathbf{p}_{2}^{\prime}\right|^{2}-\left|\mathbf{p}_{3}\right|^{2}}{\left|\mathbf{p}_{1}^{\prime}\right|\left|\mathbf{p}_{2}^{\prime}\right|^{4}\left|\mathbf{p}_{3}\right|} \\
& \times\left[\log \left(\frac{2|\mathbf{q}|}{m}\right)-2\right] .
\end{aligned}
$$

[1] P. Fulde and R. A. Ferrel, Phys. Rev. 135, A550 (1964); A. I. Larkin and Y. N. Ovchinnikov, Sov. Phys. JETP 20, 762 (1965); Y. Liao, A. S. C. Rittner, T. Paprotta, W. Li, G. B. Partridge, R. G. Hulet, S. K. Baur, and E. J. Mueller, Nature (London) 467, 567 (2010); R. Casalbuoni and G. Nardulli, Rev. Mod. Phys. 76, 263 (2004).

[2] N. Yoshida and S.-K. Yip, Phys. Rev. A 75, 063601 (2007).

[3] M. G. Alford, J. A. Bowers, and K. Rajagopal, Phys. Rev. D 63, 074016 (2001); J. A. Bowers and K. Rajagopal, Phys. Rev. D 66, 065002 (2002); D. Nickel and M. Buballa, Phys. Rev. D 79, 054009 (2009).

[4] A. W. Overhauser, Phys. Rev. Lett. 4, 462 (1960).

[5] G. Grüner, Rev. Mod. Phys. 66, 1 (1994).

[6] G. J. Conduit, A. G. Green, and B. D. Simons, Phys. Rev. Lett. 103, 207201 (2009).

[7] K. Fukushima and T. Hatsuda, Rep. Prog. Phys. 74, 014001 (2011); K. Fukushima and C. Sasaki, Prog. Part. Nucl. Phys. 72, 99 (2013).

[8] A. Schmitt, Dense Matter in Compact Stars (Springer, New York, 2011).

[9] H. Abuki, D. Ishibashi, and K. Suzuki, Phys. Rev. D 85, 074002 (2012); T. Kojo, Y. Hidaka, L. McLerran, and R. D.
Pisarski, Nucl. Phys. A843, 37 (2010); T. Kojo, Acta Phys. Pol. B Proc. Suppl. 5, 781 (2012).

[10] G. Basser and G. V. Dunne, Phys. Rev. Lett. 100, 200404 (2008); Phys. Rev. D 78, 065002 (2008); G. Basar, G. V. Dunne, and M. Thies, Phys. Rev. D 79, 105012 (2009).

[11] D. Nickel, Phys. Rev. Lett. 103, 072301 (2009); Phys. Rev. D 80, 074025 (2009).

[12] T. Tatsumi and E. Nakano, arXiv:hep-ph/0408294; E. Nakano and T. Tatsumi, Phys. Rev. D 71, 114006 (2005).

[13] R. E. Peierls, Quantum Theory of Solids (Oxford University Press, Oxford, UK, 1955).

[14] G. Grüner, Rev. Mod. Phys. 60, 1129 (1988); 66, 1 (1994).

[15] G. Grüner, Density Waves in Solids (Addison-Wesley, Reading, MA, 1999).

[16] S. Carignano, D. Nickel, and M. Buballa, Phys. Rev. D 82, 054009 (2010).

[17] D. Müller, M. Buballa, and J. Wambach, Phys. Lett. B 240, 727 (2013).

[18] S. Maedan, Prog. Theor. Phys. 123, 285 (2010).

[19] S. Karasawa and T. Tatsumi, arXiv:1307.6448.

[20] I. E. Frolov, V. Ch. Zhukovsky, and K. G. Klimenko, Phys. Rev. D 82, 076002 (2010). 


\section{QUARK BETA DECAY IN THE INHOMOGENEOUS CHIRAL ...}

PHYSICAL REVIEW D 89, 103005 (2014)

[21] T. Tatsumi, K. Nishiyama, and S. Karasawa, arXiv: 1312.0307.

[22] D. Ebert, N. V. Gubina, K. G. Klimenko, S. G. Kurbanov, and V. Ch. Zhukovsky, Phys. Rev. D 84, 025004 (2011).

[23] E. Nakano, T. Maruyama, and T. Tatsumi, Phys. Rev. D 68, 105001 (2003); M. Sadzikowski, Phys. Lett. B 553, 45 (2003); 642, 238 (2006); D. Ebert, T. G. Khunjua, K. G. Klimenko, and V. Ch. Zhukovsky, Int. J. Mod. Phys. A 29, 1450025 (2014).

[24] J. Moreira, B. Hiller, W. Broniowski, A. A. Osipov, and A. H. Blin, Phys. Rev. D 89, 036009 (2014).

[25] P. G. de Gennes and J. Prost, The Physics of Liquid Crystals (Oxford University Press, Oxford, UK, 1974).

[26] C. Kittel, Introduction to Solid State Physics (John Wiley \& Sons, New York, 1976).

[27] G. Basar, G. V. Dunne, and D. E. Kharzeev, Phys. Rev. Lett. 104, 232301 (2010).

[28] Neutron Stars and Pulsars, edited by W. Becker (Springer, New York, 2009).

[29] W. C. G. Ho and C. O. Heinke, Nature (London) 462, 71 (2009); C. O. Heinke and W. C. G. Ho, Astrophys. J. 719, L167 (2010); K. G. Elshamouty, C. O. Heinke, G. R. Sivakoff, W. C. G. Ho, P. S. Shternin, D. G. Yakovlev, D. J. Patnaude, and L. David, Astrophys. J. 777, 22 (2013).

[30] T. Noda, M. Hashimoto, N. Yasutake, T. Maruyama, T. Tatsumi, and M. Fujimoto, Astrophys. J. 765, 1 (2013).

[31] If color superconductivity develops, the phase space is exponentially restricted due to the pairing gap, so that neutrino emission is inefficient [8].
[32] N. Iwamoto, Phys. Rev. Lett. 44, 1637 (1980).

[33] The rough idea has already been presented in Ref. [34].

[34] T. Tatsumi and T. Muto, Proc. Sci., NICXII (2012) 237.

[35] O. V. Maxwell, G. E. Brown, D. K. Campbell, R. F. Dashen, and J. T. Manassah, Astrophys. J. 216, 77 (1977).

[36] T. Muto and T. Tatsumi, Prog. Theor. Phys. 80, 28 (1988).

[37] T. Tatsumi, Prog. Theor. Phys. 80, 22 (1988).

[38] This may remind us of the pion cooling [35]. The pion condensed state can be generated by a combination of global chiral rotation and local isospin rotation, but the pion momentum is supplied at the weak-interaction vertex in a similar way.

[39] L. P. Kadanoff and G. Baym, Quantum Statistical Mechanics (Perseus Books, New York, 1989).

[40] A. Sedrakian and A. E. I. Dieperrink, Phys. Rev. D 62, 083002 (2000); P. Jaikumar, C. D. Roberts, and A. Sedrakian, Phys. Rev. C 73, 042801 (2006).

[41] J. N. Bahcall and A. Wolf, Phys. Rev. 140, B1452 (1965).

[42] B. L. Friman and O. V. Maxwell, Astrophys. J. 232, 541 (1979).

[43] E. Flowers, M. Ruderman, and P. Sutherland, Astrophys. J. 205, 541 (1976); D. G. Yakovlev, A. D. Kaminker, and K. P. Levenfish, Astron. Astrophys. 343, 650 (1999).

[44] T. Maruyama, T. Muto, and T. Tatsumi (unpublished).

[45] K. Nishiyama, S. Karasawa, and T. Tatsumi (to be published).

[46] H. Sotani, T. Maruyama, and T. Tatsumi, Nucl. Phys. A906, 37 (2013). 